\title{
Quantum discord for multipartite coherent states interpolating between Werner and Greenberger-Horne-Zeilinger states
}

\author{
M. Daoud ${ }^{21}$ and R. Ahl Laamara ${ }^{b, c} 2$ \\ ${ }^{a}$ Department of Physics , Faculty of Sciences, University Ibnou Zohr, \\ Agadir, Morocco \\ ${ }^{b}$ LPHE-Modeling and Simulation, Faculty of Sciences, University Mohammed V, \\ Rabat, Morocco \\ ${ }^{c}$ Centre of Physics and Mathematics, CPM, CNESTEN, \\ Rabat, Morocco
}

\begin{abstract}
The quantum discord is used as measure of quantum correlations for two families of multipartite coherent states. The first family interpolates between generalized GHZ states and generalized Werner states. The second one is an interpolation between generalized GHZ and the ground state of the multipartite quantum system. Two inequivalent ways to split the system in a pair of qubits are introduced. The explicit expressions of quantum quantum discord in multipartite coherent states are derived. Its evaluation uses the Koashi-Winter relation in optimizing the conditional entropy. The temporal evolution of quantum correlations (quantum discord and entanglement) is also discussed.
\end{abstract}

\footnotetext{
${ }^{1}$ email: m_daoud@hotmail.com

2 email: ahllaamara@gmail.com
} 


\section{Introduction}

The characterization of quantum correlations in a bipartite or multipartite quantum system has been intensively investigated in the context of quantum information science [1, 2]. Different methods for quantifying these correlations were reported in many works (for a recent review see [3]). Entanglement, one of the prominent of quantum correlations, constitutes a typical resource to manage information in several ways 4, 5]. In this respect, a considerable attention has been paid to develop a quantitative theory of entanglement (concurrence, entanglement of formation and linear entropy [6, 7, 8, 9]). Entanglement was for a long time viewed as synonymous with quantum correlation. However, some recent studies showed that entanglement is only a special kind of quantum correlations. Indeed, unentangled quantum states can also possess quantum correlations which play a relevant role in improving quantum communication and information protocols better than their classical counter-parts [10]-15]. Therefore, as the non-classicality of correlations present in quantum states is not due solely to the presence of entanglement, there was a need of a measure to characterize and quantify the nonclassicality or quantumness of correlations which goes beyond entanglement. The first attempt in this direction was made in the works [16] and [17] where the authors concluded that when entanglement is subtracted from total quantum correlation, there remain correlations that are not entirely classical of origin. Nowadays, it is commonly accepted that the most promising candidate to measure quantum correlations is the so-called quantum discord. It has attracted considerable attention and continues to be intensively investigated in many contexts such as quantum decoherence, quantum computation and phase transition as well as other related fields (a detailed list of references can be found in [3]). Many efforts were deployed to get a closed analytical expression of quantum discord. Only some partial results were derived for some special two-qubit systems [16, 17, 18, 19]. The extension of the notion of the discord to continuous variable systems and the discussion of its properties were considered in [20]. It must be emphasized that there is now an intense recent research activity to demonstrate experimentally the advantages of the use of quantum discord without entanglement in quantum protocols. In a recent work [21], the authors experimentally show, using a variety of polarization-correlated photon pairs, non-zero quantum discord is the optimal resource for remote state preparation. More interestingly, it is found that unentangled state with non-zero quantum discord provides better performance than entangled state by achieving a higher fidelity (which is directly related to quantum discord) in remote state preparation.

In this paper we shall be mainly concerned with the pairwise quantum correlations, especially quantum discord, present in multipartite coherent states. It must be noticed that the entangled coherent states have received a special attention in the last two decades (for a recent review, see [22]). Therefore, paralleling the treatment of entanglement in coherent states systems, the main of this work is to investigate the pairwise quantum discord present in multipartite nonorthogonal states. In fact, entangled coherent states, which are typical examples of entangled nonorthogonal states, have attracted 
much attention in the literature. One may quote for instance entangled Glauber coherent states [23], $S U(2)$ and $S U(1,1)$ entangled coherent states [24]. The term entangled coherent state was introduced by Sanders in a study concerning production of entangled coherent states by using a nonlinear MachZehnder interferometer [23, 25]. Entangled coherent states were initially treated as bimodal states but later generalized to superpositions of multimode coherent states [26, 27, 28]. Generalizations to multimode systems allow the intricacies of multipartite entanglement to become manifest in entangled coherent states, as for instance in GHZ (Greenberger-Horne-Zeilinger), W (Werner) states [29, 30] and entangled coherent state versions of cluster states [31, 32, 33. In this sense, the characterization of the quantum discord in nonorthogonal multipartite states constitutes an important issue which deserves the same degree of attention as entanglement.

This paper is organized as follows. In the first section, we introduce a special instance of superpositions of multipartite coherent states. They involve Weyl-Heisenberg, $S U(2)$ and $S U(1,1)$ coherent states labeled by a single complex parameter $z$. We shall focus on balanced superpositions which are symmetric or antisymmetric under the parity transformation $z \longrightarrow-z$. The symmetric superposition is interpolating between the ground state of the multipartite system and GHZ state. The antisymmetric superposition provides a continuous interpolation between the Werner state and GHZ state. To study the bipartite quantum correlations present in the system, two different qubit mapping are considered. Section 3 is devoted to the derivation of the explicit expression of the quantum discord. The optimization over all the measurement is performed by combining the purification method and the Koashi-Winter relation . Finally, in the last section we discuss the dynamical evolution of entanglement and quantum discord under a dephasing channel. Concluding remarks close this paper.

\section{$2 \quad$ Multipartite coherent states and qubit mapping}

For several quantum systems, the coherent states can be obtained by exploiting the structure relations of the relevant dynamical group structure $G$. Therefore, any quantum state in the corresponding Hilbert space can be expanded as a sum of the coherent states associated with the group $G$. In this respect, a widespread interest was devoted to the theoretical as well experimental studies of superpositions of coherent states. Evidences of such superpositions first appeared in a study of a certain type of nonlinear Hamiltonian evolution in [34, 35]. Also, a detailed analysis concerning the manifestation of superpositions of coherent states was reported in [36, 37] (see also [38]). However, it is important to stress that superpositions of coherent states are experimentally difficult to produce,

and fundamentally this could be due to extreme sensitivity to environmental decoherence. Some experimental efforts to create superpositions of coherent states were reported in [39]. 


\subsection{Basic of coherent states}

A system of coherent states is defined to be a set $\{|z\rangle ; z \in \mathcal{D}\}$ of quantum states, in some Hilbert space $\mathcal{H}$, parameterized by the variable $z$ belonging to the set $\mathcal{D}$ such that: $z \longrightarrow|z\rangle$ is continuous and the system is over-complete; i.e.

$$
\int|z\rangle\langle z| d \mu(z)=I_{\mathcal{H}}
$$

The continuity and the resolution of the unity (1) form the minimal set of requirements to define a system of coherent states. They are not orthogonal to each other with respect to the positive measure in (11). It should be noticed that the explicit form of the coherent state $|z\rangle$ in not required for our purpose. However, to illustrate some interesting limiting situations and for the sake of completeness, we consider the familiar sets of Perelomov coherent states [40] associated with Weyl-Heisenberg, $S U(2)$ and $S U(1,1)$ groups (the extension to other groups and other coherent states definitions is straightforward). A simple way to deal with these symmetries in a unified scheme can be achieved by using the so-called generalized Weyl-Heisenbeg algebra $\mathcal{A}$ (see [41] and references therein) spanned by an annihilation operator $a^{-}$, a creation operator $a^{+}$and a number operator $N \neq a^{+} a^{-}$satisfying the structure relations

$$
\left[a^{-}, a^{+}\right]=G(N), \quad\left[N, a^{-}\right]=-a^{-}, \quad\left[N, a^{+}\right]=+a^{+},
$$

with

$$
a^{+}=\left(a^{-}\right)^{\dagger}, \quad N=N^{\dagger}, \quad G(N)=F(N+1)-F(N),
$$

where the $F$ structure function characterizes the deviation from the usual harmonic oscillator. A representation of the algebra $\mathcal{A}$ which extends that of Weyl-Heisenberg, is defined through the actions

$$
\begin{aligned}
& a^{-}|n\rangle=\sqrt{F(n)}|n-1\rangle, \quad a^{-}|0\rangle=0, \\
& a^{+}|n\rangle=\sqrt{F(n+1)}|n+1\rangle, \quad N|n\rangle=n|n\rangle
\end{aligned}
$$

on the Hilbert space spanned by the eigenvectors of $N$. This formally defines a representation of $\mathcal{A}$. Note that $a^{+} a^{-}=F(N)$, a relation that generalizes $N=a^{+} a^{-}$for the harmonic oscillator. The operator $F(N)$ can be considered as the Hamiltonian for a quantum system. This provides a physical significance to the structure function $F$. The representation (Fock-Hilbert) space $\mathcal{H}$ is generated by the orthonormal set $\{|n\rangle: n$ ranging $\}$. The dimension of the representation afforded by (44) and (5) is controlled by the positiveness of the eigenvalues $F(n)$ of the operator $F(N)$. The usual WeylHeisenberg algebra is recovered for

$$
F(N)=N
$$

The algebra $\mathcal{A}$ coincides with $s u(1,1)$ Lie algebra for

$$
F(N)=N(2 k-1+N)
$$


where the number $k$, which acquires discrete values $k=\frac{1}{2}, 1, \frac{3}{2}, 2, \ldots$, characterizes the $S U(1,1)$ discrete-series representations. The algebra $\mathcal{A}$ reproduces the spin algebra $s u(2)$ when

$$
F(N)=N(2 j+1-N)
$$

and the creation and annihilation generators coincides with the usual raising and lowering operators acting in the spin representation of dimension $2 j+1$.

The Perelomov coherent states for the algebra $\mathcal{A}$ can be constructed via a simple strategy based on the use of a Fock-Bargmann space associated with $\mathcal{A}$ [41]. This is sketched briefly as follows. Let us look for states in the form

$$
|z\rangle=\sum_{n} a_{n} z^{n}|n\rangle, \quad a_{n} \in \mathbb{R}, \quad z \in \mathbb{C}
$$

where the sum on $n$ is finite or infinite according to as $\mathcal{A}$ admits a finite- or infinite-dimensional representation. The $a_{n}$ coefficients can then be determined from the correspondence rules

$$
|n\rangle \longrightarrow a_{n} z^{n}, \quad a^{-} \longrightarrow \frac{d}{d z}
$$

applied to relations (4) and (5). This analytical realization leads to the following recurrence relation

$$
n a_{n}=\sqrt{F(n)} a_{n-1},
$$

which can be iterated to give

$$
a_{n}=\frac{\sqrt{F(n) !}}{n !}
$$

(by taking $a_{0}=1$ ). In Eq. (9), the generalized factorials are defined by

$$
F(0) !=1, \quad F(n) !=F(1) F(2) \ldots F(n) .
$$

This yields the following normalized coherent states

$$
|z\rangle=N(|z|) \sum_{n} \frac{\sqrt{F(n) !}}{n !} z^{n}|n\rangle
$$

where the normalization factor is

$$
[N(|z|)]^{-2}=\sum_{n} \frac{F(n) !}{(n !)^{2}}|z|^{2 n} .
$$

subject to convergence. They satisfy $|z\rangle=\exp \left(z a^{+}\right)|0\rangle$ and are thus coherent states in the Perelomov sense. For the usual harmonic oscillator $(F(n)=n)$, the equation (10) reads

$$
|z\rangle=e^{-\frac{|z|^{2}}{2}} \sum_{n=0}^{\infty} \frac{z^{n}}{\sqrt{n !}}|n\rangle .
$$


The overlap between two Glauber coherent states is

$$
\left\langle z_{1} \mid z_{2}\right\rangle=\exp \left(-\frac{1}{2}\left(\left|z_{1}\right|^{2}+\left|z_{2}\right|^{2}-2 \bar{z}_{1} z_{2}\right)\right)
$$

The standard set of the $S U(2)$ coherent state is obtained from (10) for $F(n)=n(2 j+1-n)$. They are given by

$$
|z\rangle=\left(1+|z|^{2}\right)^{-j} \sum_{n=0}^{2 j}\left[\frac{(2 j) !}{n !(2 j-n) !}\right]^{1 / 2} z^{n}|n\rangle .
$$

The parameter $z$ can acquire any complex value. The $S U(2)$ coherent states are normalized but they are not orthogonal to each other:

$$
\left\langle z_{1} \mid z_{2}\right\rangle=\left(1+\left|z_{1}\right|^{2}\right)^{-j}\left(1+\left|z_{2}\right|^{2}\right)^{-j}\left(1+\bar{z}_{1} z_{2}\right)^{2 j}
$$

Finally, for $F(n)=n(2 k-1+N)$, one gets the $S U(1,1)$ coherent states

$$
|z\rangle=\left(1-|z|^{2}\right)^{k} \sum_{n=0}^{\infty}\left[\frac{(2 k-1+n) !}{n !(2 k-1) !}\right]^{1 / 2} z^{n}|n\rangle .
$$

where the complex variable $z$ belongs to the unit disc $\{|z| \in \mathbb{C}, \quad|z|<1\}$. The kernel of two $S U(1,1)$ coherent states reads

$$
\left\langle z_{1} \mid z_{2}\right\rangle=\left(1-\left|z_{1}\right|^{2}\right)^{k}\left(1-\left|z_{2}\right|^{2}\right)^{k}\left(1-\bar{z}_{1} z_{2}\right)^{-2 k}
$$

\subsection{Superpositions of multipartite coherent states}

The over-completeness relation makes possible the expansion of an arbitrary state of the Hilbert space $\mathcal{H}$ in terms of the coherent states of the system under consideration. It follows that when one considers a collection of $n$ noninteracting identical particles, the whole Hilbert space is a tensor product and any multipartite coherent state can be written as a superposition of tensorial products of the form $\left|z_{1}\right\rangle \otimes\left|z_{2}\right\rangle \cdots \otimes\left|z_{n}\right\rangle \equiv\left|z_{1}, z_{2}, \cdots z_{n}\right\rangle$. In fact, the resolution of identity allows us to expand any state $|\psi\rangle$ in the space $\mathcal{H} \otimes \mathcal{H} \otimes \cdots \mathcal{H}$ as

$$
|\psi\rangle=\int d \mu\left(z_{1}\right) d \mu\left(z_{2}\right) \cdots d \mu\left(z_{n}\right)\left|z_{1}, z_{2}, \cdots z_{n}\right\rangle\left\langle z_{1}, z_{2}, \cdots z_{n} \mid \psi\right\rangle
$$

reflecting that any multipartite state can be viewed as a superposition of the coherent states $\left|z_{1}, z_{2}, \cdots z_{n}\right\rangle$. The multipartite state (17) can be reduced to a sum if the function $\psi\left(z_{1}, z_{2}, \cdots z_{n}\right)=\left\langle z_{1}, z_{2}, \cdots z_{n} \mid \psi\right\rangle$ can be expressed as a sum of delta functions. Indeed, setting

$$
\psi\left(z_{1}, z_{2}, \cdots z_{n}\right)=\left(\delta\left(z-z_{1}\right)+e^{i m \pi} \delta\left(z+z_{1}\right)\right) \delta\left(z_{1}-z_{2}\right) \delta\left(z_{2}-z_{3}\right) \cdots \delta\left(z_{n-1}-z_{n}\right),
$$

one gets the following balanced or equally weighted superposition of multipartite coherent states

$$
|\psi\rangle \equiv|z, m, n\rangle=\mathcal{N}\left(|z\rangle \otimes|z\rangle \otimes \cdots \otimes|z\rangle+e^{i m \pi}|-z\rangle \otimes|-z\rangle \otimes \cdots \otimes|-z\rangle\right)
$$

where $m \in \mathbb{Z}$. The normalization factor $\mathcal{N}$ is

$$
\mathcal{N}=\left[2+2 p^{n} \cos m \pi\right]^{-1 / 2}
$$


where $p$ denotes the overlap between the states $|z\rangle$ and $|-z\rangle$. It is given

$$
p=\langle z \mid-z\rangle .
$$

It is real as it can be verified from (10). For Weyl-Heisenberg, $S U(2)$ and $S U(1,1)$ coherent states, the quantity $p$ is obtainable from the expressions (12), (14) and (16), respectively. Two interesting limits of superpositions of the form (18) arise when $p \rightarrow 0$ and $p \rightarrow 1$.

We first consider the asymptotic limit $p \rightarrow 0$. In this limit the two states $|z\rangle$ and $|-z\rangle$ approach orthogonality, and an orthogonal basis can be constructed such that $|\mathbf{0}\rangle \equiv|z\rangle$ and $|\mathbf{1}\rangle \equiv|-z\rangle$. Thus, the state $|z, m, n\rangle$ approaches a multipartite state of GHZ type

$$
|z, m, n\rangle \sim|\mathrm{GHZ}\rangle_{n}=\frac{1}{\sqrt{2}}\left(|\mathbf{0}\rangle \otimes|\mathbf{0}\rangle \otimes \cdots \otimes|\mathbf{0}\rangle+e^{i m \pi}|\mathbf{1}\rangle \otimes|\mathbf{1}\rangle \otimes \cdots \otimes|\mathbf{1}\rangle\right) .
$$

In the situation where $p \rightarrow 1$ (or $z \rightarrow 0)$, one should distinguish separately the cases $m=0(\bmod 2)$ and $m=1(\bmod 2)$. For $m$ even, the multipartite superposition (18) reduces to ground state

$$
|0,0(\bmod 2), n\rangle \sim|0\rangle \otimes|0\rangle \otimes \cdots \otimes|0\rangle
$$

and for $m$ odd, the state $|z, 1(\bmod 2), n\rangle$ reduces to a multipartite state of Werner type [42]

$$
|0,1(\bmod 2), n\rangle \sim|\mathrm{W}\rangle_{n}=\frac{1}{\sqrt{n}}(|1\rangle \otimes|0\rangle \otimes \cdots \otimes|0\rangle+|0\rangle \otimes|1\rangle \otimes \ldots \otimes|0\rangle+\cdots+|0\rangle \otimes|0\rangle \otimes \cdots \otimes|1\rangle) .
$$

Here $|n\rangle(n=0,1)$ denote the Fock-Hilbert states.

It follows that the states $|z, m=0(\bmod 2), n\rangle$ interpolate between states of GHZ type $(p \rightarrow 0)$ and the separable state $|0\rangle \otimes|0\rangle \otimes \cdots \otimes|0\rangle(p \rightarrow 1)$. In other hand, the states $|z, m=1(\bmod 2), n\rangle$ may be viewed as interpolating between states of GHZ type $(p \rightarrow 0)$ and states of Werner type $(p \rightarrow 1)$.

To close this subsection, it is important to emphasize that the main concern in investigating the properties of superpositions of coherent states is how to produce such states. Their experimental production is fundamentally difficult to achieve. This is especially due to extreme sensitivity to environmental decoherence. Experimental efforts to create superpositions of coherent states with the present day technology, are encouraging. Indeed, superpositions of weak coherent states with opposite phase, resembling to a small "Schrödinger's cat" state (or "Schrödinger's kitten"), were produced by photon subtraction from squeezed vacuum [43. Also, the experimental generation of arbitrarily large squeezed Schrodinger cat states, using homodyne detection and photon number states (two photons) as resources was reported in [43]. Very recently, creation of coherent state superpositions, by subtracting up to three photons from a pulse of squeezed vacuum light, is reported in [44. The mean photon number of such coherent states produced by three-photon subtraction is of 2:75. The production of cat states especially ones of high amplitude or mean number of photons remains an experimental challenge. Considering the fast technical progress and the increasing number of groups working in this field, we expect that the generation of cat states (and Bell states) is a goal that is achievable in the near future. 


\subsection{Qubit mapping}

\subsubsection{Pure states}

To study the bipartite quantum correlations present in (18), the whole system can be partitioned in two different ways. We first consider bipartite splitting of the multipartite system, i.e., splitting the entire system into two subsystems, one subsystem containing any $k(1 \leq k \leq n-1)$ particles and the other containing the remaining $n-k$ particles. Accordingly, one writes the state $|z, m, n\rangle$ as

$$
|z, m, n\rangle=\mathcal{N}\left(|z\rangle_{k} \otimes|z\rangle_{n-k}+e^{i m \pi}|-z\rangle_{k} \otimes|-z\rangle_{n-k}\right)
$$

where

$$
| \pm z\rangle_{l}=| \pm z\rangle_{1} \otimes| \pm z\rangle_{2} \otimes \cdots \otimes| \pm z\rangle_{l}, \quad l=k, n-k .
$$

The multi-particle state $|z, m, n\rangle$ can be expressed as a state of two logical qubits. For this end, we introduce, for the first subsystem, the orthogonal basis $\left\{|0\rangle_{k},|1\rangle_{k}\right\}$ defined as

$$
|0\rangle_{k}=\frac{|z\rangle_{k}+|-z\rangle_{k}}{\sqrt{2\left(1+p^{k}\right)}} \quad|1\rangle_{k}=\frac{|z\rangle_{k}-|-z\rangle_{k}}{\sqrt{2\left(1-p^{k}\right)}} .
$$

Similarly, we introduce, for the second subsystem, the orthogonal basis $\left\{|0\rangle_{n-k},|1\rangle_{n-k}\right\}$ given by

$$
|0\rangle_{n-k}=\frac{|z\rangle_{n-k}+|-z\rangle_{n-k}}{\sqrt{2\left(1+p^{n-k}\right)}} \quad|1\rangle_{n-k}=\frac{|z\rangle_{n-k}-|-z\rangle_{n-k}}{\sqrt{2\left(1-p^{n-k}\right)}} .
$$

Reporting the equations (23) and (24) in (22), one has the explicit form of the pure state $|z, m, n\rangle$ in the basis $\left\{|0\rangle_{k} \otimes|0\rangle_{n-k},|0\rangle_{k} \otimes|1\rangle_{n-k},|1\rangle_{k} \otimes|0\rangle_{n-k},|1\rangle_{k} \otimes|1\rangle_{n-k}\right\}$. It is given by

$$
|z, m, n\rangle=\sum_{\alpha=0,1} \sum_{\beta=0,1} C_{\alpha, \beta}|\alpha\rangle_{k} \otimes|\beta\rangle_{n-k}
$$

where the coefficients $C_{\alpha, \beta}$ are

$$
\begin{array}{ll}
C_{0,0}=\mathcal{N}\left(1+e^{i m \pi}\right) a_{k} a_{n-k}, & C_{0,1}=\mathcal{N}\left(1-e^{i m \pi}\right) a_{k} b_{n-k} \\
C_{1,0}=\mathcal{N}\left(1-e^{i m \pi}\right) a_{n-k} b_{k}, & C_{1,1}=\mathcal{N}\left(1+e^{i m \pi}\right) b_{k} b_{n-k} .
\end{array}
$$

in term of the quantities

$$
a_{l}=\sqrt{\frac{1+p^{l}}{2}}, \quad b_{l}=\sqrt{\frac{1-p^{l}}{2}} \quad \text { for } l=k, n-k
$$

involving the overlap $p$ between two coherent states of equal amplitude and opposite phase.

\subsubsection{Mixed states}

The second partition can be realized by considering the bipartite reduced density matrix $\rho_{k l}$ which is obtained by tracing out all other subsystems except ones labeled by the indices $k$ and $l$. There are $n(n-1) / 2$ different density matrices $\rho_{k l}$. It is simple to see that all the reduced density matrices $\rho_{k l}$ are identical. Therefore, it is sufficient to consider $\rho_{12}$ and to generalize from this case. Then, by 
tracing out systems $3,4, \ldots, n$ in the state $|z, m, n\rangle$, we obtain the reduced density matrix describing the particles or modes 1 and 2 as

$$
\begin{aligned}
\rho_{12} & =\operatorname{Tr}_{3,4, \ldots, n}(|z, m, n\rangle\langle z, m, n|) \\
& =\mathcal{N}^{2}\left(|z, z\rangle\langle z, z|+|-z,-z\rangle\left\langle-z,-z\left|+e^{i m \pi} q\right|-z,-z\right\rangle\left\langle z, z\left|+e^{-i m \pi} q\right| z, z\right\rangle\langle-z,-z|\right)
\end{aligned}
$$

with $q \equiv p^{n-2}$. To study the correlations of the system described by the density matrix $\rho_{12}$, we convert it into a two-qubit system. Thus, we choose an orthogonal pair $\{|\mathbf{0}\rangle,|\mathbf{1}\rangle\}$ as

$$
|z\rangle \equiv a|\mathbf{0}\rangle+b|\mathbf{1}\rangle,|-z\rangle \equiv a|\mathbf{0}\rangle-b|\mathbf{1}\rangle,
$$

where

$$
a=\sqrt{\frac{1+p}{2}} \quad b=\sqrt{\frac{1-p}{2}} .
$$

The logical qubits $|\mathbf{0}\rangle$ and $|\mathbf{1}\rangle$ given by

$$
|\mathbf{0}\rangle=\frac{1}{\sqrt{2+2 p}}(|z\rangle+|-z\rangle) \quad|\mathbf{1}\rangle=\frac{1}{\sqrt{2-2 p}}(|z\rangle-|-z\rangle) .
$$

coincide with even and odd coherent states, respectively. Substituting the equation (27) into (26), we obtain the density matrix

$$
\rho_{12}=\mathcal{N}^{2}\left(\begin{array}{cccc}
2 a^{4}(1+q \cos m \pi) & 0 & 0 & 2 a^{2} b^{2}(1+q \cos m \pi) \\
0 & 2 a^{2} b^{2}(1-q \cos m \pi) & 2 a^{2} b^{2}(1-q \cos m \pi) & 0 \\
0 & 2 a^{2} b^{2}(1-q \cos m \pi) & 2 a^{2} b^{2}(1-q \cos m \pi) & 0 \\
2 a^{2} b^{2}(1+q \cos m \pi) & 0 & 0 & 2 b^{4}(1+q \cos m \pi)
\end{array}\right)
$$

in the basis $\{|\mathbf{0 0}\rangle,|\mathbf{0 1}\rangle,|\mathbf{1 0}\rangle,|\mathbf{1 1}\rangle\}$. It is remarkable that the obtained density belongs to the set of the so-called $X$ states. It can be also written, in the Bloch representation, as

$$
\rho_{12}=\sum_{\alpha=0}^{3} \sum_{\beta=0}^{3} R_{\alpha, \beta} \sigma^{\alpha} \otimes \sigma^{\beta}
$$

where the correlation matrix $R$ is given by

$$
R=\left(\begin{array}{cccc}
1 & 0 & 0 & 2 \mathcal{N}^{2} p(1+q \cos m \pi) \\
0 & 2 \mathcal{N}^{2}\left(1-p^{2}\right) & 0 & 0 \\
0 & 0 & -2 \mathcal{N}^{2}\left(1-p^{2}\right) q \cos m \pi & 0 \\
2 \mathcal{N}^{2} p(1+q \cos m \pi) & 0 & 0 & 2 \mathcal{N}^{2}\left(p^{2}+q \cos m \pi\right)
\end{array}\right)
$$

and $\sigma^{0}$ and $\sigma^{i}(i=1,2,3)$ stand for identity and the usual Pauli matrices, respectively.

\section{Quantifying the quantum discord}

So far, quantum discord has been calculated explicitly only for a rather limited set of two-qubit quantum states and analytical expressions for more general quantum states are not known. In this section, we give another instance of states for which quantum discord can be evaluated explicitly. Indeed, we shall derive the explicit form of this kind of bipartite correlation in the state (18) by making use of the qubit mapping corresponding to the partitioning schemes (22) and (26) discussed in the previous section. 


\subsection{Definitions}

The quantum discord is defined as the difference between total correlation and classical correlation. The total correlation is usually quantified by the mutual information $I$

$$
I\left(\rho_{A B}\right)=S\left(\rho_{A}\right)+S\left(\rho_{B}\right)-S\left(\rho_{A B}\right)
$$

where $\rho_{A B}$ is the state of a bipartite quantum system composed of the subsystems $A$ and $B$, the operator $\rho_{A(B)}=\operatorname{Tr}_{B(A)}\left(\rho_{A B}\right)$ is the reduced state of $A(B)$ and $S(\rho)$ is the von Neumann entropy of a quantum state $\rho$. Suppose that a positive operator valued measure (POVM) measurement is performed on subsystem $A$. The set of POVM elements is denoted by $\mathcal{M}=\left\{M_{k}\right\}$ with $M_{k} \geqslant 0$ and $\sum_{k} M_{k}=\mathbb{I}$. In this paper, we deal with two-qubit rank two states (with only two nonzero eigenvalues). In this case, the generalized positive operator valued measurement is not required. Indeed, it has been clearly shown in [45, 46] (see also the recent review [47]) that for a bipartite mixed state of two qubits of rank two , the optimal measurement giving the quantum discord is a two element POVM. The elements of such POVM are orthogonal projectors. Thus, it is natural to consider only projective measurements for the subsystem $A$. The von Neumann measurement (from now on just a measurement) on the subsystem $A$ project the system into a statistical ensemble $\left\{p_{B, k}, \rho_{B, k}\right\}$ such that

$$
\rho_{A B} \longrightarrow \frac{\left(M_{k} \otimes \mathbb{I}\right) \rho_{A B}\left(M_{k} \otimes \mathbb{I}\right)}{p_{B, k}}
$$

where the measurement operation is written as [18]

$$
M_{k}=U \Pi_{k} U^{\dagger}
$$

with $\Pi_{k}=|k\rangle\langle k|(k=0,1)$ is the projector for subsystem $A$ along the computational base $|k\rangle$, $U \in S U(2)$ is a unitary operator and

$$
p_{B, k}=\operatorname{tr}\left[\left(M_{k} \otimes \mathbb{I}\right) \rho_{A B}\left(M_{k} \otimes \mathbb{I}\right)\right] .
$$

The amount of information acquired about particle $B$ is then given by

$$
S\left(\rho_{B}\right)-\sum_{k} p_{B, k} S\left(\rho_{B, k}\right),
$$

which depends on measurement $\mathcal{M}$. This dependence can be removed by doing maximization over all the measurements, which gives rise to the definition of classical correlation

$$
\begin{gathered}
C\left(\rho_{A B}\right)=\max _{\mathcal{M}}\left[S\left(\rho_{B}\right)-\sum_{k} p_{B, k} S\left(\rho_{B, k}\right)\right] \\
=S\left(\rho^{B}\right)-\widetilde{S}_{\min }
\end{gathered}
$$

where $\widetilde{S}_{\text {min }}$ denotes the minimal value of the conditional entropy

$$
\widetilde{S}=\sum_{k} p_{B, k} S\left(\rho_{B, k}\right)
$$


It follows that quantum discord is then given by

$$
D\left(\rho_{A B}\right)=I\left(\rho_{A B}\right)-C\left(\rho_{A B}\right)=S\left(\rho_{A}\right)+\widetilde{S}_{\min }-S\left(\rho_{A B}\right) .
$$

The main step in evaluating the quantum discord is the minimization of conditional entropy to get an explicit expression of the quantum discord in the multipartite system (18).

\subsection{Quantum discord for pure bipartite coherent states}

According to the first partitioning scheme (22), the entire $n$ particles system is split into two components $A$, containing $k$ particles, and $B$, containing $n-k$ particles. The bipartite density state $\rho_{k, n-k}=|z, m, n\rangle\langle z, m, n|$ is pure and the conditional density is also a pure state. This implies that the quantum conditional entropy is zero. Then, the quantum discord for the pure state $\rho_{A B} \equiv \rho_{k, n-k}$ coincides with the von Neumann entropy of the subsystem $A$ :

$$
D\left(\rho_{k, n-k}\right)=S\left(\rho_{k}\right)
$$

where $\rho_{k}$ is the reduced density of the subsystem $A$. In this scheme the quantum discord can be computed easily. It is given by

$$
D\left(\rho_{k, n-k}\right)=-\lambda_{+} \log _{2} \lambda_{+}-\lambda_{-} \log _{2} \lambda_{-}
$$

in term of the eigenvalues of the reduced density matrix $\rho_{k}$ given by

$$
\lambda_{ \pm}=\frac{1}{2}\left(1 \pm \sqrt{1-\mathcal{C}_{k, n-k}^{2}}\right)
$$

where $\mathcal{C}_{k, n-k}$ is the concurrence between the subsystems $A$ and $B$ :

$$
\mathcal{C}_{k, n-k}=2\left|C_{0,0} C_{1,1}-C_{1,0} C_{0,1}\right|=\frac{\sqrt{1-p^{2 k}} \sqrt{1-p^{2(n-k)}}}{1+p^{n} \cos m \pi}
$$

where we used the mapping (25). Note that the entanglement of formation given by

$$
E\left(\rho_{k, n-k}\right)=H\left(\frac{1}{2}+\frac{1}{2} \frac{p^{k}+p^{n-k} \cos m \pi}{1+p^{n} \cos m \pi}\right)
$$

is nothing but the von Neumann entropy of the subsystem $A$. Here $H$ stands for the binary entropy $H(x)=h(x)+h(1-x)$ with $h(x)=-x \log _{2} x$. Hence, we have the following closed relation between quantum discord and entanglement of formation

$$
D\left(\rho_{k, n-k}\right)=E\left(\rho_{k, n-k}\right)
$$

This agrees with the fact that quantum discord and entanglement of formation are identical for pure states and amount to the same set of correlations.

In the limiting case $p \rightarrow 0$, the state (22) is of GHZ type having a maximal bipartite entanglement $\left(\mathcal{C}_{k, n-k}=1\right)$ and we obtain

$$
D\left(\rho_{k, n-k}\right)=1
$$


The situation becomes different when $p \rightarrow 1$. In this case, we have

$$
D\left(\rho_{k, n-k}\right)=0
$$

for symmetric pure states (i.e. $m$ even) as expected (in this limit the state (18) is a $n$ tensorial product of the ground state $|0\rangle$ ). In the limit $p \rightarrow 1$, the antisymmetric states (i.e. $m$ odd) are of Werner type. The bipartite concurrence is

$$
\mathcal{C}_{k, n-k}=\frac{2}{n} \sqrt{k(n-k)},
$$

and the corresponding pairwise quantum discord takes the form

$$
D\left(\rho_{k, n-k}\right)=H\left(\frac{1}{2}+\frac{1}{2} \frac{|n-2 k|}{n}\right) .
$$

The quantum correlations (entanglement and quantum discord) in multipartite Werner states vanish as $n$ becomes large.

\subsection{Quantum discord for mixed bipartite coherent states}

\subsubsection{Mutual information entropy}

The subsystems $A$ and $B$ of the subsection 3.1 correspond here to the modes or particles 1 and 2 described by the density operator $\rho_{12}(29)$. The non vanishing eigenvalues of the density matrix $\rho_{12}$ are

$$
\lambda_{ \pm}=\frac{1}{2} \frac{\left(1 \pm p^{2}\right)(1 \pm q \cos (m \pi))}{1+p^{n} \cos (m \pi)}
$$

and the joint entropy is

$$
S\left(\rho_{12}\right)=h\left(\lambda_{+}\right)+h\left(\lambda_{-}\right)=H\left(\lambda_{+}\right) .
$$

The eigenvalues of the marginal $\rho_{1}=\operatorname{Tr}_{2} \rho_{12}$ are

$$
\lambda_{1, \pm}=\frac{1}{2} \frac{(1 \pm p)(1 \pm p q \cos (m \pi))}{1+p^{n} \cos (m \pi)},
$$

and the marginal entropy reads

$$
S\left(\rho_{1}\right)=h\left(\lambda_{1,+}\right)+h\left(\lambda_{1,-}\right)=H\left(\lambda_{1,+}\right) .
$$

The eigenvalues of the marginal $\rho_{2}=\operatorname{Tr}_{1} \rho_{12}$ are

$$
\lambda_{2, \pm}=\frac{1}{2} \frac{(1 \pm p)(1 \pm p q \cos (m \pi))}{1+p^{n} \cos (m \pi)},
$$

and the corresponding entropy is given by

$$
S\left(\rho_{2}\right)=h\left(\lambda_{2,+}\right)+h\left(\lambda_{2,-}\right)=H\left(\lambda_{2,+}\right) .
$$

Note that for the mixed density under consideration the marginal densities $\rho_{1}$ and $\rho_{2}$ are identical. The explicit form of the mutual information (32) writes

$$
I\left(\rho_{12}\right)=2 H\left(\frac{1}{2} \frac{(1+p)\left(1+p^{n-1} \cos (m \pi)\right)}{1+p^{n} \cos (m \pi)}\right)-H\left(\frac{1}{2} \frac{\left(1+p^{2}\right)\left(1+p^{n-2} \cos (m \pi)\right)}{1+p^{n} \cos (m \pi)}\right) .
$$




\subsubsection{Conditional entropy}

After computing the quantum mutual information, we next compute the classical correlation $C\left(\rho_{12}\right) \equiv$ $C\left(\rho_{A B}\right)$. This requires an explicit expression of the unitary operator $U$ occurring in (33). So, we parameterizes $U$ as follows

$$
U=\exp \left(\eta \sigma_{+}-\bar{\eta} \sigma_{-}\right) \exp \left(i \phi \sigma_{3}\right) \quad \eta \in \mathbb{C}, \phi \in \mathbb{R}
$$

Using this parametrization, one can verify that the quantities defined by

$$
\left\langle\sigma_{i}\right\rangle_{k}=\left\langle k\left|U^{\dagger} \sigma_{i} U\right| k\right\rangle, \quad i=1,2,3 \quad \text { and } \quad k=0,1
$$

are given by

$$
\left\langle\sigma_{3}\right\rangle_{k}=(-)^{k} \frac{1-\bar{\alpha} \alpha}{1+\bar{\alpha} \alpha}, \quad\left\langle\sigma_{1}\right\rangle_{k}=(-)^{k} \frac{\bar{\alpha}+\alpha}{1+\bar{\alpha} \alpha} \quad,\left\langle\sigma_{2}\right\rangle_{k}=i(-)^{k} \frac{\bar{\alpha}-\alpha}{1+\bar{\alpha} \alpha}
$$

where $\alpha=-i \frac{\eta}{\sqrt{\bar{\eta} \eta}} \tan \sqrt{\bar{\eta} \eta}$. They can be also written as

$$
\left\langle\sigma_{3}\right\rangle_{k}=(-)^{k} \cos \theta, \quad\left\langle\sigma_{1}\right\rangle_{k}=(-)^{k} \sin \theta \cos \varphi \quad,\left\langle\sigma_{2}\right\rangle_{k}=(-)^{k} \sin \theta \sin \varphi
$$

where $\frac{\theta}{2} e^{i \varphi}=-i \eta$. The conditional density operators $\rho_{B, k} \equiv \rho_{2, k}$ are

$$
\rho_{2, k}=\frac{1}{p_{2, k}}\left(\begin{array}{cc}
\left(1+R_{03}\right)+\left(R_{30}+R_{33}\right)\left\langle\sigma_{3}\right\rangle_{k} & R_{11}\left\langle\sigma_{1}\right\rangle_{k}-i R_{22}\left\langle\sigma_{2}\right\rangle_{k} \\
R_{11}\left\langle\sigma_{1}\right\rangle_{k}+i R_{22}\left\langle\sigma_{2}\right\rangle_{k} & \left(1-R_{03}\right)+\left(R_{30}-R_{33}\right)\left\langle\sigma_{3}\right\rangle_{k}
\end{array}\right)
$$

where the matrix elements $R_{\alpha \beta}$ are giving by (31) and

$$
p_{2, k}=\frac{1}{2}\left(1+R_{30}\left\langle\sigma_{3}\right\rangle_{k}\right) .
$$

It follows that the conditional entropy given by

$$
\widetilde{S}=\sum_{k=0,1} p_{2, k} S\left(\rho_{2, k}\right)
$$

can be cast in the following form

$$
\widetilde{S}=\sum_{k=0,1} p_{2, k} H\left(\frac{1}{2}+\frac{1}{2} \sqrt{1-4 \operatorname{det} \rho_{2, k}}\right) .
$$

Evidently, the quantities $p_{2, k}$ and $\operatorname{det} \rho_{2, k}$ can be expressed in terms of the angular directions $\theta$ and $\varphi$ by making use of equations (49), (150) and (151). Consequently, we can explicitly write $\widetilde{S}=\widetilde{S}(\theta, \varphi)$ and perform the minimization over the azimuthal and polar angles $\theta$ and $\varphi$. However, there exists another elegant method to perform such an optimization. This is presented in the following subsection. 


\subsubsection{Minimization of conditional entropy}

To minimize the conditional entropy (53), we shall use the purification method and the Koashi-Winter relation [48] (see also [49]). This relation establishes the connection between the classical correlation of a bipartite state $\rho_{A B} \equiv \rho_{12}$ and the entanglement of formation of its complement $\rho_{B C} \equiv \rho_{23}$. This connection will be clarified hereafter. The density $\rho_{12}(29)$ is a two-qubit state of rank two. It decomposes as

$$
\rho_{12}=\lambda_{+}\left|\phi_{+}\right\rangle\left\langle\phi_{+}\left|+\lambda_{-}\right| \phi_{-}\right\rangle\left\langle\phi_{-}\right|
$$

where the eigenvalues $\lambda_{+}$and $\lambda_{-}$are given by (43) and the corresponding eigenstates $\left|\phi_{+}\right\rangle$and $\left|\phi_{-}\right\rangle$ are given by

$$
\left|\phi_{+}\right\rangle=\frac{1}{\sqrt{2\left(1+p^{2}\right)}}[(1+p)|\mathbf{0}, \mathbf{0}\rangle+(1-p)|\mathbf{1}, \mathbf{1}\rangle] \quad\left|\phi_{-}\right\rangle=\frac{1}{\sqrt{2}}[|\mathbf{0}, \mathbf{1}\rangle+|\mathbf{1}, \mathbf{0}\rangle]
$$

in the basis (28). Attaching a qubit 3 to the two-qubit system 1 and 2, we write the purification of $\rho_{12}$ as

$$
|\phi\rangle=\sqrt{\lambda_{1}}\left|\phi_{+}\right\rangle \otimes|\mathbf{0}\rangle+\sqrt{\lambda_{-}}\left|\phi_{-}\right\rangle \otimes|\mathbf{1}\rangle
$$

such that the whole system 123 is described by the pure density state $\rho_{123}=|\phi\rangle\langle\phi|$ from which one has the bipartite densities $\rho_{12}=\operatorname{Tr}_{3} \rho_{123}$ and $\rho_{23}=\operatorname{Tr}_{1} \rho_{123}$.

Suppose now that a von Neumann measurement $\left\{M_{0}, M_{1}\right\}$ is performed on the subsystem 1 (here also we need positive operator valued measurement of rank one that is proportional the one dimensional projector). From the viewpoint of the whole system in the pure state $|\phi\rangle$, the measurement gives rise to an ensemble for $\rho_{23}$ that we denote by

$$
\mathcal{E}_{23}=\left\{p_{k},\left|\phi_{23, k}\right\rangle\right\}
$$

where

$$
p_{k}=\left\langle\psi\left|M_{k} \otimes \mathbb{I} \otimes \mathbb{I}\right| \psi\right\rangle \quad, \quad\left|\phi_{23, k}\right\rangle\left\langle\phi_{23, k}\right|=\frac{1}{p_{k}} \operatorname{Tr}_{1}\left[\left(M_{k} \otimes \mathbb{I} \otimes \mathbb{I}\right)|\psi\rangle\langle\psi|\right] .
$$

On the other hand, from the viewpoint of the state $\rho_{12}$, the von Neuman measurement on 1 gives rise to the ensemble for $\rho_{2}$ that it was defined previously as $\mathcal{E}_{2}=\left\{p_{2, k}, \rho_{2, k}\right\}$ (note that $p_{k}=p_{2, k}$ ). It is simple to check that the ensemble $\mathcal{E}_{2}$ can be induced from $\mathcal{E}_{23}$ by tracing out the qubit 3, namely

$$
\rho_{2, k}=\operatorname{Tr}_{3}\left[\left|\phi_{23, k}\right\rangle\left\langle\phi_{23, k}\right|\right] .
$$

We denote by $E\left(\left|\phi_{23, k}\right\rangle\right)$ the measure of entanglement for pure states. It is given by the von Neumann entropy of the reduced subsystem $\rho_{2, k}=\operatorname{Tr}_{3}\left(\left|\phi_{23, k}\right\rangle\left\langle\phi_{23, k}\right|\right)$

$$
E\left(\left|\phi_{23, k}\right\rangle\right)=S\left(\rho_{2, k}\right)
$$

It follows that the average of entanglement of formation over the ensemble $\mathcal{E}_{23}$

$$
\bar{E}_{23}=\sum_{k=0,1} p_{k} E\left(\left|\phi_{23, k}\right\rangle\right)
$$


coincides with the conditional entropy (53). At this point, it is important to note that Koachi and Winter have pointed out that the minimum value of $\bar{E}_{23}$ is exactly the entanglement of formation of $\rho_{23}$. Consequently, the minimal value of the conditional entropy coincides with the entanglement of formation of $\rho_{23}$ :

$$
\widetilde{S}_{\min }=E\left(\rho_{23}\right)
$$

which is easy to evaluate. Indeed, we get

$$
\widetilde{S}_{\min }=E\left(\rho_{23}\right)=H\left(\frac{1}{2}+\frac{1}{2} \sqrt{1-\left|C\left(\rho_{23}\right)\right|^{2}}\right)
$$

where the concurrence of the density $\rho_{23}$ is

$$
\left|C\left(\rho_{23}\right)\right|^{2}=\frac{p^{2}\left(1-p^{2}\right)\left(1-p^{2 n-4}\right)}{\left(1+p^{n} \cos m \pi\right)^{2}}
$$

Using the equation (53), one can verify the relation

$$
\widetilde{S}_{\min }=\widetilde{S}\left(\theta=\frac{\pi}{2}, \phi=0\right)
$$

which reflects that the minimal value of conditional entropy is reached for $\theta=\frac{\pi}{2}$ and $\phi=0$. Finally, reporting (44), (45) and (58) in the definition (36), the explicit expression of quantum discord for the density $\rho_{12}$ is

$$
\begin{array}{r}
D\left(\rho_{12}\right)=H\left(\frac{1}{2} \frac{(1+p)\left(1+p^{n-1} \cos (m \pi)\right)}{1+p^{n} \cos (m \pi)}\right)-H\left(\frac{1}{2} \frac{\left(1+p^{2}\right)\left(1+p^{n-2} \cos (m \pi)\right)}{1+p^{n} \cos (m \pi)}\right) \\
\left.+H\left(\frac{1}{2}+\frac{1}{2} \sqrt{1-\frac{p^{2}\left(1-p^{2}\right)\left(1-p^{2 n-4}\right)}{\left(1+p^{n} \cos m \pi\right)^{2}}}\right)\right)
\end{array}
$$

in term of the the overlap $p$. Clearly, this result can be also derived using the method developed (independently) in [19] and [50] (see also [51]) to obtain the quantum discord for some special instances of the so-called $X$ states. However, one should stress that our method based on the purification trick combined with the Koashi-Winter relation reduce drastically the optimization process of the conditional entropy. It provides a direct relation between quantum discord and entanglement of formation reflecting how these correlations are distributed in a given tripartite pure system. It has been used in several papers dealing with quantum correlations as for instance in [52] where the authors emphasized its crucial role in exploring the distribution of entanglement in the deterministic quantum computation with one single pure qubit and a collection of an arbitrary number of mixed states.

\subsubsection{Some particular cases}

We start with the special case $n=2$. The state (26) is pure and $q=1$. The quantum discord (59)) reads

$$
D\left(\rho_{12}\right)=H\left(\frac{(1+p)(1+p \cos m \pi)}{2\left(1+p^{2} \cos m \pi\right)}\right)
$$


It coincides with the quantum discord $D\left(\rho_{k, n-k}\right)$ given by the equation (41) for $n=2$ and $k=1$. In particular, for symmetric states ( $m$ even), one obtains

$$
D\left(\rho_{12}\right)=D\left(\rho_{1,1}\right)=H\left(\frac{(1+p)^{2}}{2\left(1+p^{2}\right)}\right)
$$

and $D\left(\rho_{12}\right)=D\left(\rho_{1,1}\right)=1$ for antisymmetric states ( $m$ odd) as shown in the figure 1 .

Here also, it is interesting to consider the limiting cases $p \rightarrow 0$ and $p \rightarrow 1$ as it was done previously for pure states. For $n>2$, the quantum discord vanishes (59) when $p \rightarrow 0$. It vanishes also when $p \rightarrow 1$ for $m$ even. However, for $m$ odd, the quantum discord (59) reduces to the special form

$$
D\left(\rho_{12}\right)=H\left(1-\frac{1}{n}\right)+H\left(\frac{1}{2}+\frac{1}{2} \frac{\sqrt{n^{2}-4 n+8}}{n}\right)-H\left(1-\frac{2}{n}\right)
$$

and goes to zero for $n$ large.

To corroborate our analysis, we give in the figures 2 and 3 the behavior of the quantum discord (59). Figure 2 gives a plot of quantum discord versus the overlap $p$ for the mixed state $\rho_{12}$ with $m$ even (the symmetric case). As seen from the figure, after an initial increasing, the quantum discord decreases to vanish when $p \rightarrow 1$. The maximum of quantum discord depends on the number of particles contained in the system. It is remarkable that for $n=25$ the maximum is larger than ones obtained for $n=4$ and $n=5$. Beside the numerical results reported in the figure 2, we have also studied the behavior of quantum discord for other values of $n$. This study shows that for $6 \leq n \leq 25$ the maximum of quantum discord is greater than one reached for $n=5$. Also, the behavior of quantum discord for $n \geq 20$ is very close to the case $n=25$ presented in the figure 2 . In figure 3 , we give a plot of the quantum discord (59) for $m$ odd (the antisymmetric case) and different values of $n$. In this case the quantum discord increases as $p$ increases for a small number of particles and the maximal value of quantum discord is reached for $p \rightarrow 1$. However, for a higher number of particles $n(n=25$ for instance), the maximum is reached for $p<1$. In the limit $p \rightarrow 1$, we have a Werner state $|W\rangle_{n}$ and the pairwise quantum discord given by (60) decreases to vanish in the limit of a large number of particles $(n \rightarrow \infty)$. For $p \rightarrow 0$, corresponding to GHZ type states, the quantum discord is zero.

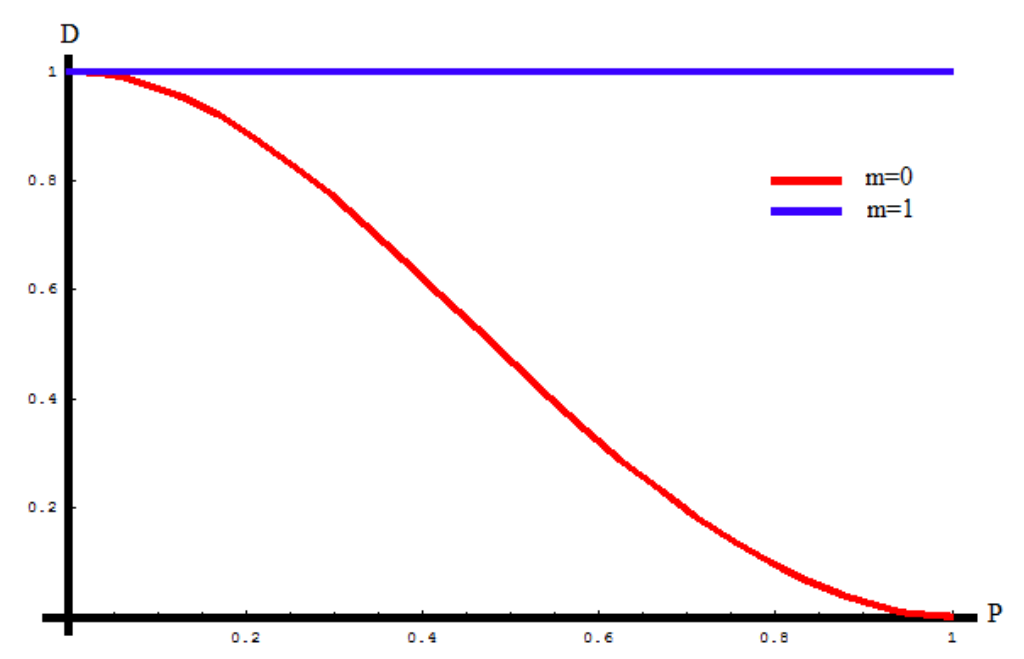

FIG. 1: The pairwise quantum discord $D$ versus the overlap $p$ for $n=2$. 


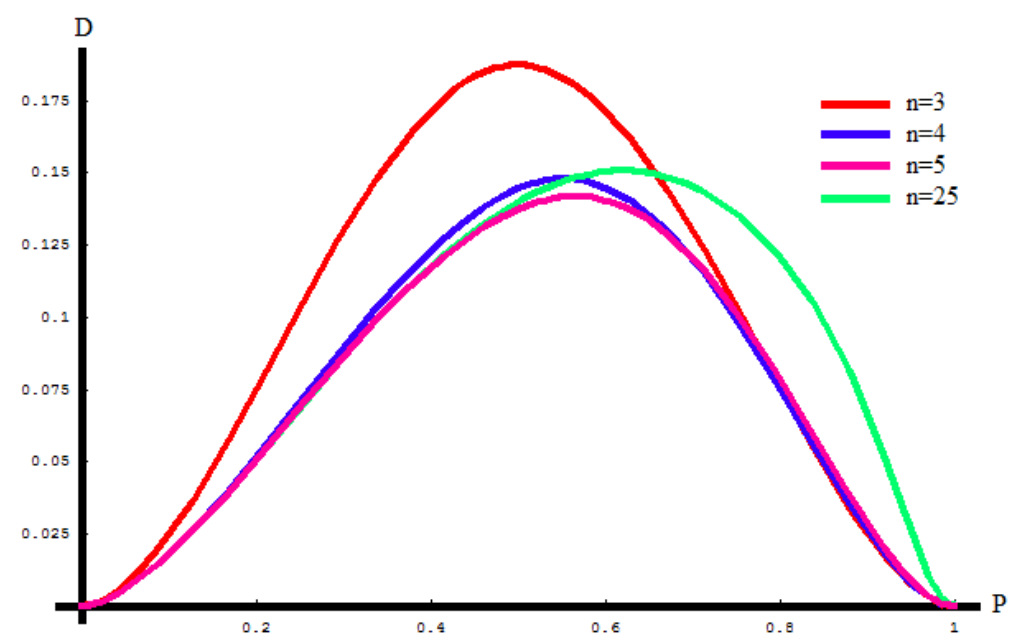

FIG. 2: The pairwise quantum discord $D$ versus the overlap $p$ for symmetric states.

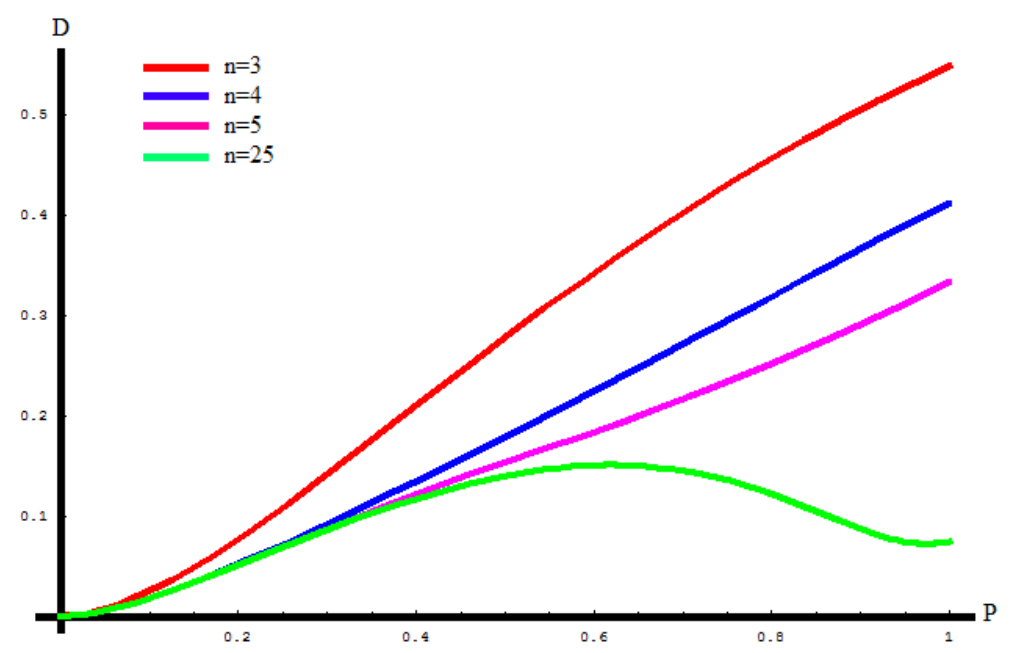

FIG. 3: The pairwise quantum discord $D$ versus the overlap $p$ for anti-symmetric states.

\section{Dynamics of quantum correlations under dephasing channel}

The sudden disappearance of entanglement is one of the most intriguing features in quantum mechanics. In fact, it has been observed that in a pair of entangled qubits, interacting with noisy environments, entanglement can disappear in a finite time [53]. This phenomenon, termed in the literature "entanglement sudden death", was experimentally confirmed [54].

In this section, we investigate the dynamics of bipartite quantum correlations (entanglement and quantum discord) of the multipartite coherent states $|z, m, n\rangle(n>2)$. We focus on the second partitioning scheme (26) where mixed states emerge. The quantum discord and entanglement are two different correlations for mixed states (contrarily to the pure case). We consider the evolution of a mixed bipartite system under a dephasing dissipative channel. In this order, we use the Kraus operator approach [4] which describes conveniently the dynamics of two qubits interacting independently with individual environments. The time evolution of the bipartite density $\rho_{12} \equiv \rho_{12}(0)$ (29) can be written compactly 
as

$$
\rho_{12}(t)=\sum_{\mu, \nu} E_{\mu, \nu}(t) \rho_{12}(0) E_{\mu, \nu}^{\dagger}(t)
$$

where the so-called Kraus operators

$$
E_{\mu, \nu}(t)=E_{\mu}(t) \otimes E_{\nu}(t) \quad \sum_{\mu, \nu} E_{\mu, \nu}^{\dagger} E_{\mu, \nu}=\mathbb{I} .
$$

The operators $E_{\mu}$ describe the one-qubit quantum channel effects. The non-zero Kraus operators for a dephasing channel are given by

$$
E_{0}=\operatorname{diag}(1, \sqrt{1-\gamma}) \quad E_{1}=\operatorname{diag}(0, \sqrt{\gamma})
$$

with $\gamma=1-e^{-\Gamma t}$ and $\Gamma$ denoting the decay rate. It is easy to check that the density matrix (29) evolves as

$$
\rho_{12}(t)=\mathcal{N}^{2}\left(\begin{array}{cccc}
2 a^{4}(1+q \cos m \pi) & 0 & 0 & 2(1-\gamma) a^{2} b^{2}(1+q \cos m \pi) \\
0 & 2 a^{2} b^{2}(1-q \cos m \pi) & 2(1-\gamma) a^{2} b^{2}(1-q \cos m \pi) & 0 \\
0 & 2(1-\gamma) a^{2} b^{2}(1-q \cos m \pi) & 2 a^{2} b^{2}(1-q \cos m \pi) & 0 \\
2(1-\gamma) a^{2} b^{2}(1+q \cos m \pi) & 0 & 0 & 2 b^{4}(1+q \cos m \pi)
\end{array}\right)
$$

Using the prescription provided in the works [55] and [56] to measure the amount of entanglement in bipartite quantum states, one can check that the concurrence is given by

$$
C(t)=2 \max \left\{0, \Lambda_{1}(t), \Lambda_{2}(t)\right\}
$$

where

$$
\begin{aligned}
& \Lambda_{1}(t)=2 \mathcal{N}^{2} a^{2} b^{2}[(1-\gamma)(1+q \cos m \pi)-(1-q \cos m \pi)] \\
& \Lambda_{2}(t)=2 \mathcal{N}^{2} a^{2} b^{2}[(1-\gamma)(1-q \cos m \pi)-(1+q \cos m \pi)]
\end{aligned}
$$

It follows that the concurrence is given by

$$
C(t)=\frac{1}{2} \frac{1-p^{2}}{1+p^{n} \cos m \pi}\left[e^{-\Gamma t}\left(1+p^{n-2}\right)-\left(1-p^{n-2}\right)\right]
$$

for

$$
t<t_{0}=\frac{1}{\Gamma}\left[\ln \left(1+p^{n-2}\right)-\ln \left(1-p^{n-2}\right)\right]
$$

and the system is entangled. However, for $t>t_{0}$, the concurrence is zero and the entanglement disappears, i.e. the system is separable. This clearly reflects that under dephasing channel, the entanglement suddenly vanishes. Note that the bipartite system under consideration is initially (in the absence of an external interaction) entangled. Indeed, for $t=0$, the concurrence is

$$
C(0)=\frac{p^{n-2}-p^{n}}{1+p^{n} \cos m \pi}
$$

and is always non zero except in the limiting cases $p \rightarrow 0$ or $p \rightarrow 1$ for $m$ even. Notice that for $m$ odd, the concurrence is zero for $p \rightarrow 0$ but does not vanish when $p \rightarrow 1$ and it is given by $2 / n$. 
It is important to stress that the quantum discord $D\left(\rho_{12}(0)\right)$ is nonzero except in the particular case $p \rightarrow 0$. To show that the quantum discord does not disappear after the interaction of the system with the dissipative channel, we note that the density matrix $\left.\rho_{12}(t)\right)$ belongs to the class of the socalled circulant states [57]. Thus, one can use the vanishing quantum discord criteria, discussed in [57], to check that the state $\rho_{12}(t)$ has vanishing quantum discord if and only if $p \rightarrow 0$. This implies that even when entanglement suddenly disappears in a finite time, quantum discord does not vanish. This agrees with the commonly accepted fact that the quantum discord is more robust than entanglement to sudden death under a dissipative channel.

\section{$5 \quad$ Concluding remarks}

In this paper, we have obtained explicit expressions of quantum discord for symmetric and antisymmetric superpositions of multipartite coherent states. These states cover the generalized GHZ and generalized Werner states. In particular, the balanced antisymmetric superpositions ( $m$ odd) interpolate continuously between generalized GHZ and generalized Werner states. The key point in determining the bipartite quantum correlations is based on the splitting of the entire system in two qubit subsystems. Two inequivalent splitting schemes were discussed. The first one leads to a pure bipartite density and the quantum discord coincides with the entanglement of formation. The second consists in constructing bipartite systems by a trace procedure keeping only the modes in which we are interested. In this way, mixed states are obtained and the corresponding quantum discord was explicitly derived. This derivation requires an optimization over all the measurement needed to extract the amount of quantum correlation which is general difficult to perform. To avoid such a difficulty, we used the purification method together with the Koashi-Winter relation which are advantageous in simplifying the minimization process of the conditional entropy. In the last part of the paper, we discussed the robustness of the quantum discord present in multipartite coherent states in comparison with the entanglement. We have shown that in sending the system through a dephasing channel the entanglement can be lost. This is not the case of quantum discord which behaves more robust against dissipative channels. It must be noticed that the results obtained here can be extended easily to many other classes of coherent states even those associated with higher symmetries which are labeled by several variables. Finally, we stress that the bipartite correlation does not capture genuine multipartite correlations. It follows that it is interesting to investigate the measure of genuine multipartite quantum discord for arbitrary multipartite non orthogonal states in the spirit of the results recently obtained in [58].

\section{References}

[1] R. Horodecki, P. Horodecki, M. Horodecki and K. Horodecki, Rev. Mod. Phys. 81(2009) 865.

[2] O. Gühne and G. Tóth, Phys. Rep. 474 (2009) 1. 
[3] K. Modi, A. Brodutch, H. Cable, T. Paterek and V. Vedral, Quantum discord and other measures of quantum correlation, arXiv:1112.6238.

[4] M.A. Nielsen and I.L. Chuang, Quantum Computation and Quantum Information (Cambridge Univ. Press, Cambridge, 2000).

[5] V. Vedral, Rev. Mod. Phys. 74 (2002) 197.

[6] P. Rungta, V. Buzek, C.M. Caves, M. Hillery and G.J. Milburn, Phys. Rev. A 64 (2001) 042315.

[7] C.H. Bennett, D.P. DiVincenzo, J. Smolin and W.K. Wootters, Phys. Rev. A 54 (1996) 3824.

[8] W.K. Wootters, Phys. Rev. Lett. 80 (1998) 2245.

[9] V. Coffman, J. Kundu and W.K. Wootters, Phys. Rev. A 61 (2000) 052306.

[10] E. Knill and R. Laflamme, Phys. Rev. Lett. 81 (1998) 5672.

[11] S.L. Braunstein, C.M. Caves, R. Jozsa, N. Linden, S. Popescu and R. Schack, Phys. Rev. Lett. 83 (1999) 1054.

[12] C.H. Bennett, D.P. DiVincenzo, C.A. Fuchs, T. Mor, E. Rains, P.W. Shor, J.A. Smolin and W.K. Wootters, Phys. Rev. A 59 (1999) 1070.

[13] D.A. Meyer, Phys. Rev. Lett. 85 (2000) 2014.

[14] E. Biham, G. Brassard, D. Kenigsberg and T. Mor, Theor. Comput. Sci. 320 (2004) 15.

[15] A. Datta, S.T. Flammia and C.M. Caves, Phys. Rev. A 72, 042316 (2005); A. Datta and G. Vidal, ibid. 75(2007) 042310; A. Datta, A. Shaji and C.M. Caves, Phys. Rev. Lett. 100 (2008) 050502 .

[16] L. Henderson and V. Vedral, J. Phys. A 34(2001) 6899; V. Vedral, Phys. Rev. Lett. 90 (2003) 050401; J. Maziero, L. C. Celéri, R.M. Serra and V. Vedral, Phys. Rev A 80 (2009) 044102.

[17] H. Ollivier and W.H. Zurek, Phys. Rev. Lett. 88 (2001) 017901.

[18] S. Luo, Phys. Rev. A 77 (2008) 042303; Phys. Rev. A 77 (2008) 022301.

[19] M. Ali, A.R.P. Rau and G. Alber, Phys. Rev. A 81 (2010) 042105.

[20] P. Giorda and M. G. A. Paris, Phys. Rev. Lett. 105 (2010) 020503.

[21] B. Dakic, Y.O. Lipp, X. Ma, M. Ringbauer, S. Kropatschek, S. Barz, T. Paterek, V. Vedral, A. Zeilinger, C. Brukner and P. Walther, Quantum discord as optimal resource for quantum communication, arXiv:1203.1629 (2012).

[22] B.C Sanders, J. Phys. A: Math. Theor. 45 (2012) 244002. 
[23] B.C. Sanders, Phys. Rev. A 45 (1992) 6811.

[24] X. Wang, B. C. Sanders and S. H. Pan, J. Phys. A 33 (2000) 7451.

[25] B.C. Sanders, Phys. Rev. A 46 (1992) 2966.

[26] I. Jex, P. Törmä and S. Stenholm, J. Mod. Opt. 42 (1995) 1377.

[27] S.-B. Zheng, Quant. Semiclass. Opt. B: J. European Opt. Soc. B 10 (1998) 691.

[28] X. Wang and B.C. Sanders, Phys. Rev. A 65 (2001) 012303.

[29] H. Jeong and N. B. An, Phys. Rev. A 74 (2006) 022104.

[30] H.-M. Li, H.-C. Yuan and H.-Y. Fan, Int. J. Theor. Phys. 48 (2009) 2849.

[31] P. P. Munhoz, F. L. Semião and Vidiello, Phys. Lett. A 372 (2008) 3580.

[32] W.-F. Wang, X.-Y. Sun and X.-B. Luo, Chin. Phys. Lett. 25(2008) 839.

[33] E.M. Becerra-Castro, W.B. Cardoso, A.T. Avelar and B. Baseia, J. Phys. B: At. Mol. Opt. Phys. 41 (2008) 085505.

[34] G.J. Milburn, Phys. Rev. A 33 (1985) 674.

[35] G.J. Milburn and C.A. Holmes, Phys. Rev. Lett. 56 (1986) 2237.

[36] B. Yurke and D. Stoler, Phys. Rev. Lett. 57 (1986) 13.

[37] B. Yurke and D. Stoler, Phys. Rev. A 35 (1987) 4846.

[38] V. Buz̃ek and P. L. Knight, Progress in Optics 34 (1995) 1.

[39] M. Brune, E. Hagley, J. Dreyer, X. Maitre, A. Maali, C. Wunderlich, J. M. Raimond and S. Haroche, Phys. Rev. Lett. 77 (1996) 4887.

[40] A. Perelomov, Generalized Coherent States and Their Applications, Springer, Berlin, 1986.

[41] M. Daoud and M. Kibler, Bosonic and k-fermionic coherent states for a class of polynomial Weyl-Heisenberg algebras, arXiv:1110.4799, To appear in J. Phys. A (2012).

[42] W. Dür, G. Vidal and J.I. Cirac, Phys. Rev. A 62 (2000) 062314.

[43] A. Ourjoumtsev, H. Jeong, R. Tualle-Brouri and P. Grangier, Nature 448 (2007) 784.

[44] T. Gerrits, S. Glancy, T.S. Clement, B. Calkins, A.E. Lita, A.J. Miller, A. L. Migdall, S.W. Nam, R.P. Mirin and E. Knill, Phys. Rev. A 82 (2010) 031802(R).

[45] S. Hamieh, R. Kobes and H. Zaraket, Phys. Rev. A 70 (2004) 052325. 
[46] F. Galve, G. Giorgi and R. Zambrini, EPL 96 (2011) 40005

[47] K. Modi, A. Brodutch, H. Cable, T. Paterek and V. Vedral, Quantum discord and other measures of quantum correlation, arXiv:1112.6238,

[48] M. Koachi and A. Winter, Phys. Rev. A 69 (2004) 022309.

[49] M. Shi, W. Yang, F. Jiang and J. Du, J. Phys. A: Math. Theor. 44 (2011) 415304.

[50] F.F. Fanchini, T. Werlang, C.A. Brasil, L.G.E. Arruda and A.O. Caldeira, Phys. Rev. A. 81 (2010) 052107.

[51] B. Li, Z-X Wang and S-M Fei, Phys. Rev. A 83 (2011) 022321.

[52] F.F. Fanchini, M.F. Cornelio, M.C. de Oliveira and A.O. Caldeira, Phys. Rev. A 84 (2011) 012313.

[53] T. Yu and J.H. Eberly, Phys. Rev. Lett. 97 (2006) 140403.

[54] M.P. Almeida, F. de Melo, M. Hor-Meyll, A. Salles, S.P. Walborn, P.H. Souto Ribeiro and L. Davidovich, Science 316 (2007) 579.

[55] W.K. Wootters, Phys. Rev. Lett. 80 (1998) 2245; W.K. Wootters, Quant. Inf. Comp. 1 (2001) 27.

[56] S. Hill and W.K. Wootters, Phys. Rev. Lett. 78 (1997) 5022.

[57] B. Bylicka, D. Chruściński, Circulant states with vanishing quantum discord, arXiv:1104.1804.

[58] Z-H. Ma and Z-H. Chen, Witness for a measure of genuine multipartite quantum discord for arbitrary $N$ partite quantum state, arXiv:1108.4323 